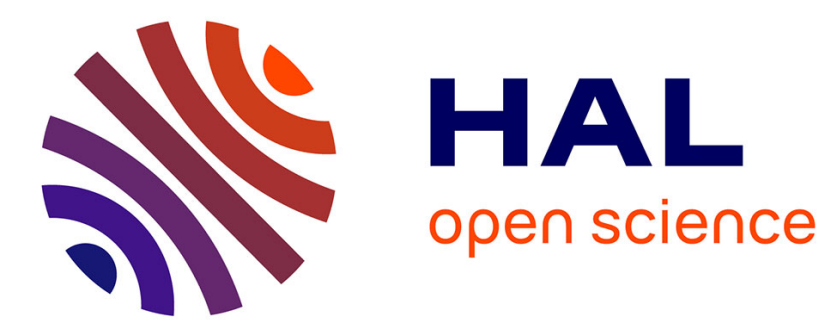

\title{
VU D'AILLEURS : LA FABRIQUE POPULAIRE DE LA VILLE
}

Fanny Gerbeaud, Sylvain Grisot, Caroline Motta, Lenka Sobotová

\section{To cite this version:}

Fanny Gerbeaud, Sylvain Grisot, Caroline Motta, Lenka Sobotová. VU D'AILLEURS: LA FABRIQUE POPULAIRE DE LA VILLE. Revue Projet, 2015. hal-02434615

\section{HAL Id: hal-02434615 https://hal.science/hal-02434615}

Submitted on 16 Jan 2020

HAL is a multi-disciplinary open access archive for the deposit and dissemination of scientific research documents, whether they are published or not. The documents may come from teaching and research institutions in France or abroad, or from public or private research centers.
L'archive ouverte pluridisciplinaire HAL, est destinée au dépôt et à la diffusion de documents scientifiques de niveau recherche, publiés ou non, émanant des établissements d'enseignement et de recherche français ou étrangers, des laboratoires publics ou privés. 


\title{
VU D'AILLEURS : LA FABRIQUE POPULAIRE DE LA VILLE
}

\author{
Fanny Gerbeaud, Sylvain Grisot, Caroline Motta et Lenka Sobotová
}

\author{
C.E.R.A.S | « Revue Projet»
}

$2015 / 5 \mathrm{~N}^{\circ} 348$ | pages 57 à 67

ISSN 0033-0884

Article disponible en ligne à l'adresse :

https://www.cairn.info/revue-projet-2015-5-page-57.htm

Distribution électronique Cairn.info pour C.E.R.A.S.

(C) C.E.R.A.S. Tous droits réservés pour tous pays.

La reproduction ou représentation de cet article, notamment par photocopie, n'est autorisée que dans les limites des conditions générales d'utilisation du site ou, le cas échéant, des conditions générales de la licence souscrite par votre établissement. Toute autre reproduction ou représentation, en tout ou partie, sous quelque forme et de quelque manière que ce soit, est interdite sauf accord préalable et écrit de l'éditeur, en dehors des cas prévus par la législation en vigueur en France. Il est précisé que son stockage dans une base de données est également interdit. 


\section{Vud'ailleurs : la fabrique populaire de la ville}

Formes spontanées d'urbanisation, les bidonvilles interrogent le travail des urbanistes et le rôle des autorités. Mais les réponses diffèrent. Illustrations en Thaïlande, en Colombie et dans le département français de Mayotte.

\section{«Communautés denses » à Bangkok}

Fanny Gerbeaud est architecte et docteure en sociologie. Enseignante à l'école d'architecture de Bordeaux, elle a consacré sa thèse de doctorat aux pratiques spontanées de production de l'espace dans la métropole de Bangkok, en Thaïlande.

- n Thaïlande comme dans nombre de pays émergents, la ville - est partiellement construite par ses habitants, souvent les plus précaires. Cette urbanisation informelle, face à la ville planifiée, est loin d'être marginale. On la retrouve dans les bidonvilles, que les Thaïs nomment " communautés denses ", mais aussi dans les multiples extensions illégales opérées sur des immeubles, notamment de logements sociaux. 
Les bidonvilles, qui abritaient près de $20 \%$ des habitants de Bangkok ${ }^{1}$ en 2000, sont l'expression d'une urbanisation spontanée traditionnelle. Avant l'adoption des titres de propriété et du système cadastral, la population vivait dans des habitations en matériaux périssables (en bois, en bambou), sur l'eau ou sous forme de hameaux. Plus anciennes que la capitale elle-même, certaines communautés denses abritent parfois des architectures locales et un artisanat traditionnel. La plupart se trouvent aujourd'hui enserrées par les constructions récentes et menacées d'expulsion. Même si tous les foyers ne sont pas pauvres, et que certains vivent depuis plusieurs générations à Bangkok, la mise à niveau des habitations et la reconnaissance de ce mode d'urbanisation sont le préalable à leur stabilisation. Il est vain de penser contrôler l'ensemble des constructions spontanées. En tirer, au contraire, des enseignements sur leur rapport à la ville et leur potentiel s'impose désormais chez les décideurs et chez les professionnels de l'espace.

\section{DU BIDONVILLE AU QUARTIER RÉSIDENTIEL AUTOGÉRÉ}

Les communautés denses participent étroitement au bon fonctionnement métropolitain, logeant des foyers peu aisés et assurant une maind'œuvre et des biens bon marché. Elles s'adaptent en permanence aux contraintes spatiales, économiques et géographiques. À proximité des nombreux canaux, les constructions témoignent d'une autre manière de concevoir l'habitat, plus en lien avec le climat et certaines traditions locales (croyances liées à l'eau, pisciculture, navigation...). Les logements s'assimilent au tissu urbain au fil du temps, notamment dans les communautés régularisées : le bâti rénové et agrandi tend à rattraper la production du marché privé jusqu'à adopter les standards et l'esthétique du bâti pavillonnaire. Les ruelles s'élargissent pour se connecter aux voies publiques (...). Parfois les transports en commun et des services municipaux viennent achever l'ouverture de ces nouveaux quartiers et encourager une fréquentation extérieure. Il ne s'agit donc plus de lieux de relégation voués à la destruction, mais bien de quartiers résidentiels en devenir. Certaines communautés denses sont aujourd'hui attractives pour des classes moyennes

1. $19 \%$ en 2000, selon Pornchokchai Sopon, Global report on human settlements 2003, City report, Bangkok, 2003. 
en quête d'alternative aux tours résidentielles. D'anciens hameaux occupent désormais des emplacements stratégiques en plein quartier d'affaires. Et progressivement, la vision des Bangkokiens sur ces quartiers évolue. Dans les zones à forte pression foncière, la qualité de vie et la végétation priment sur le stigmate du bidonville pour les employés ou cadres qui s'y implantent. D'autres qui ont grandi sur place occupent des postes de responsabilité à proximité : rester sur place est pour eux l'affirmation d'un attachement à des liens de voisinage et à un environnement.

\section{PRÉSERVER LES COMMUNAUTÉS}

Les communautés denses constituent également des réseaux d'entraide pour les primo-arrivants venus de la campagne ou des pays voisins. Quand elles sont menacées d'expulsion, les communautés tendent à se fédérer en réseaux et à mettre au point des projets et des initiatives pour favoriser leur régularisation. Elles placent leurs actions dans la perspective de l'administration métropolitaine : journées de dépollution des canaux, traitement des déchets et des eaux usées, mise aux normes des voies pour faciliter la circulation fluviale et l'accès aux secours... Des réunions sont organisées entre communautés pour diffuser les initiatives réussies et guider celles qui peinent à obtenir leur régularisation.

La Thailande dispose de plusieurs formes de régularisation. Certaines permettent le maintien sur site des habitants et, surtout, de leur structure communautaire. Elles sont encadrées par l'institut Codi ${ }^{2}$, qui met à disposition des " architectes de communauté », des crédits à faible taux, et intervient pour régler les questions foncières auprès des décideurs. La régularisation collective réduit le risque de spéculation foncière : les liens communautaires et les espaces partagés sont davantage préservés. Chaque habitant participe au remboursement du prêt et à la gestion communautaire. En cas de départ d'un foyer, le logement demeure abordable en retournant à la communauté. Les habitants engagent ainsi la rénovation en co-conception avec les architectes, et participent aux travaux en relation étroite avec les autorités de district.

2. L'institut pour le développement des organisations communautaires, le Codi (Community organizations development institute), est un organisme semi-public. II est financé par le remboursement des micro-crédits octroyés aux premières communautés. 
Certes la communauté régularisée doit respecter les normes du district, mais les habitants y trouvent la légitimation de leur communauté dans la ville. La co-conception est une réelle reconnaissance de leur compétence dans la fabrication d'un morceau de ville, ainsi que dans leur capacité à le gérer ensemble, à long terme.

Ces bouleversements ont une portée politique, en ce sens qu'ils démocratisent la fabrication et la gestion de la ville. Cette construction de la ville par des actions individuelles, cumulées au fil du temps, est source de profondes évolutions chez les professionnels de l'espace. Ceux-ci délèguent une partie de leurs compétences à la population.

\section{GREFFES SPONTANÉES SUR LOGEMENTS SOCIAUX}

Les professionnels prennent aussi appui sur les transformations faites par les habitants pour optimiser la production planifiée du logement et améliorer la qualité de vie. Prenons l'exemple des grands ensembles de logements sociaux de Bangkok, construits par l'Office national du logement (NHA) dès les années 1960. Les résidents y ont profondément transformé l'architecture moderne, de l'intérieur des logements exigus aux vastes espaces extérieurs alors inexploités. Les anciens bidonvillois y ont "greffé " des éléments à des structures en béton pour les adapter à leurs usages et à leurs désirs. Ces ajouts traduisent une appropriation des espaces de transition (entre le logement et la rue) avec le retour des activités commerciales et professionnelles dans le parc public de logements sociaux. Car le paiement mensuel du loyer et des charges, bien que peu élevés, requiert pour plusieurs foyers le maintien d'une activité d'appoint, souvent informelle.

Les services de proximité le long des principales artères du parc social assurent la jonction avec les rues alentour et créent un couloir commercial convivial. Les cantines et stands mobiles de vente alimentaire jouxtent les cages d'escalier. Entre les immeubles ou sur les aires engazonnées, des résidents jardinent de petites surfaces ou aménagent des salons d'extérieur. Les balcons, refermés par des grilles en surplomb, deviennent une pièce de stockage ou une cuisine d'extérieur. Les débordements sur coursives ou directement sur la voie transforment, enfin, les appartements en maisons avec garage et accès privé. 


\section{VERS UNE CONSTRUCTION PARTAGÉE DE LA VILLE}

Ces " greffes " sur un parc planifié sont un moyen de retourner une situation peu favorable et de mieux lier des ensembles d'architecture moderne au reste de la ville. Elles rompent la monumentalité des barres de logements. Elles recréent aussi des repères pour les anciens bidonvillois : la fabrication d'espaces partagés, de cuisines de plein air, l'individualisation des logements ou le retour de points d'alimentation.

D'abord interdites par le gestionnaire, ces modifications se voient désormais encadrées par des baux très avantageux : le bailleur (la NHA) propose des emplacements extérieurs afin d'en réguler le développement. Les nouveaux ensembles de logements sociaux proposent désormais des surfaces d'activité commerciale et professionnelle à destination des habitants, mais aussi des logements plus diversifiés. La NHA confirme ainsi son rôle social (...).

Les transformations des logements sociaux sont révélatrices des lacunes de la production planifiée. Elles témoignent aussi de la coexistence possible d'une urbanisation spontanée aux côtés de celle des " sachants ".

\section{QUAND LA POPULATION CRÉE SON CADRE DE VIE}

Déléguer une part de la construction urbaine aux habitants, que ce soit des populations précaires ou les habitants du pavillonnaire français en cours de densification, n'est pas nécessairement risquer la dégradation du cadre bâti. La situation thaïlandaise montre au contraire que ces actions créent un point de rencontre entre «nonsachants » et professionnels, pour un possible partage de compétences et un développement urbain concerté. À Bangkok, les assouplissements réglementaires s'assortissent, finalement, d'un renforcement du rôle des praticiens de l'urbain. Plus en prise avec les aspirations et les contraintes des habitants de communautés denses, ils gagnent en légitimité à mesure qu'ils composent la ville à plusieurs mains. Ce type de démarches intéresse aussi de nombreux jeunes professionnels en France. Elles pourraient s'appliquer à la rénovation des grands ensembles ou permettre de réduire les coûts de production du logement social en donnant le droit aux locataires de personnaliser leur habitat. 


\section{Mayotte : cachez ce quartier que je ne saurais voir}

Sylvain Grisot est intervenu pendant plusieurs années dans les bidonvilles de Mayotte en tant qu'anthropologue et urbaniste. Il a depuis fondé l'agence de projets dixit.net, qui s'engage aux côtés des acteurs de la ville dans les projets de transformation du tissu urbain existant.

'accession de Mayotte au statut de département d'outre-mer (Dom) en 2011 pourrait laisser croire à une normalisation de sa situation au sein de l'outre-mer français, mais elle reste la plus africaine des îles de la République. (...) Largement perfusée par la métropole, l'île a connu un développement extrêmement rapide depuis la fin des années 70, qui a creusé l'écart avec le reste de l'archipel des Comores. Baisse de la mortalité ${ }^{3}$, transition démographique inachevée ${ }^{4}$, immigration clandestine massive ${ }^{5}$... Autant de facteurs qui ont fait exploser son peuplement. Mayotte a ainsi vu sa population tripler depuis 1985, pour dépasser les 210000 habitants en 2012 (+2,7 \% par an sur l'ensemble de l'île, un taux dépassant les 8 \% sur certaines communes).

Cette croissance, dont la rapidité est difficilement appréhendable depuis la métropole, s'accompagne d'une expansion très rapide de la tache urbaine autour des principales agglomérations. Si rapide qu'elle déborde le rythme de développement des infrastructures, et rend obsolètes les plans d'aménagement avant qu'ils n’entrent en vigueur ${ }^{6}$.

\section{LE DUR ET LE MOU}

Les bidonvilles ont désormais conquis les pentes abruptes de cette île volcanique et les franges des mangroves à quelques mètres du lagon, au mépris des risques naturels multiples qui les menacent régulièrement. Les constructions en terre, bambou et feuilles de

3. Le taux de mortalité est passé de 25\%o habitants en 1958 à 7,36 \%o en 2008.

4. L'indice synthétique de fécondité restait de 4,1 enfants par femme en 2012 contre 8,1 en 1978.

5. Selon les estimations officielles, entre 25 et $30 \%$ de la population de Mayotte était constituée d'étrangers en situation irrégulière en 2013.

6. Il n'y a par nature pas de données exhaustives sur la population des bidonvilles à l'échelle de lîle. Les seules données fiables sont issues des enquêtes de terrain menées sur certains quartiers, toujours différentes et difficiles à agréger. Il est toutefois certain que plusieurs dizaines de milliers de personnes sont concernées. 
palmier ont cédé depuis longtemps la place aux tôles ondulées; la précarité et l'insalubrité s'amplifient avec l'extension de ces quartiers qui regroupent souvent plusieurs centaines d'habitants (...).

Si la transformation de cette ville spontanée en ville organisée est amorcée, le processus n'est ni doux ni linéaire. Symbolisée par le passage du bâti en "dur ", par opposition au " mou " de la tôle et des planches, la mutation du bidonville en morceau de ville est un processus de mise aux normes qui transforme aussi le peuplement de ces quartiers, au-delà de leur seule forme bâtie.

Le processus de "durcification ", engagé essentiellement par l'État, est aussi volontariste qu'impuissant. Menée uniquement sur les rares secteurs officiellement dédiés à l'urbanisation, la résorption de l'insalubrité nie de fait l'existence d'une urbanisation massive des pentes et des secteurs à risque. Là où elle est amorcée, la durcification permet la légalisation des occupations foncières, l'aménagement et l'assainissement des quartiers, voire la mutation du bâti par la construction de logements sociaux attribués à quelques-uns (...).

Cette mutation prend du temps - souvent trop -, mais elle permet d'intégrer à la ville organisée certains quartiers spontanés, en s'inspirant parfois de l'intelligence des trames ambiguës de ces morceaux de ville opportunément construits sans urbanistes.

\section{AMÉLIORER PLUTÔT QUE METTRE AUX NORMES}

Mais ce processus de durcification ne résout pas les problèmes, il les déplace. Concentrées sur les seuls secteurs où l'urbanisation est légalisable, les politiques de l'État sont aussi ciblées sur les seuls habitants de ces quartiers qui ont une existence reconnue : elles repoussent ailleurs l'essentiel de la population des bidonvilles, qui s'organise pour amorcer l'urbanisation illégale sur de nouvelles pentes. Plus loin des regards...

Cet abandon d'une part du territoire et de sa population est le fruit d'une double ambiguïté de l'intervention urbaine. D'abord, une attitude légaliste et hygiéniste fonde toute intervention sur l'impérieuse nécessité de mettre aux normes, quitte à abandonner à leur sort des quartiers qui ne pourront pas prendre part à la ville légale. Pas d'intervention a minima, pas d'améliorations graduelles, et finalement aucune alternative entre l'inaction et l'intervention massive (...). Et pourtant il faudrait intervenir dans ces quartiers : créer des postes 
avancés permettant d'offrir des équipements sanitaires et des lieux pour accueillir permanences sociales et médicales, créer des accès pour les véhicules de secours, stabiliser les secteurs érodés, sécuriser les ravines, conseiller les habitants pour améliorer la sécurité des bâtis, réguler l'usage du foncier, lutter contre les marchands de sommeil, anticiper le risque cyclonique...

La seconde ambiguïté est sans doute liée au regard confus porté sur ces bidonvilles par la profession de l'urbanisme. Le bidonville est le symbole inacceptable de la ville non finie, il est par nature une étape de transition : une étape de la ville qui se durcit, une étape de la vie de ses habitants qui passent par ce seuil pour accéder au monde urbain. Il faudrait enfin admettre que l'intervention urbaine ne vise pas à créer la ville parfaite, finie, mais à accompagner un processus, le guider, l'améliorer. Elle doit être à l'écoute du réel, de ce qu'est la ville et non pas de ce qu'elle devrait être.

\section{Colombie : quand le bidonville interroge les urbanistes}

\section{Caroline Motta et Lenka Sobotová}

Caroline Motta, architecte, docteure en urbanisme, est membre du conseil d'administration de la Société française des urbanistes. Elle développe à Bogotá des recherches liées à l'aménagement du territoire. Lenka Sobotová, géographe, est enseignante à l'université Externado de Colombie.

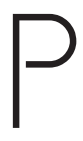

lus ou moins visibles à ras du sol selon la configuration d'un territoire, les bidonvilles occupent une partie conséquente de l'aire métropolitaine des villes latino-américaines. En juin 2015, pour mieux comprendre cette informalité urbaine, nous avons rencontré plusieurs acteurs de la ville colombienne, à Bogotá et à Cali. Bogotá logeait 700000 habitants en 1951, et environ huit millions en 2015. Cali est passée de 100000 habitants en 1940 à presque trois millions aujourd'hui. La majorité des arrivants sont des paysans, d'abord au chômage suite à l'industrialisation de l'agriculture, puis déplacés en masse ces quinze dernières années par les conflits armés qui secouent le pays. Très vite, l'afflux a dépassé la capacité d'accueil traditionnelle 
des villes et la capacité d'action des responsables de l'aménagement. L'insolvabilité des nouveaux habitants les oblige à occuper de manière illégale des terrains considérés comme inutilisables (...).

\section{DE L'ÎLOT AU QUARTIER}

(...) Carlos M. est actuellement concierge d'un immeuble d'appartements dans le nord de Bogotá. C'est la deuxième fois qu'il fabrique sa maison de ses propres mains, avec des matériaux de recyclage (bidons métalliques, bois) et achetés (tuiles en zinc), sans fenêtres. D’abord sur un terrain "d'invasion » (occupé sans aucune règle ni démarche), puis en achetant par quotas une parcelle à un " urbanisateur " pirate, "propriétaire » d'un terrain (illégal) dans l'arrondissement de Ciudad Bolívar, la plus grande concentration d'habitat populaire et informel au sud de la capitale. Aujourd'hui, le lotissement où il habite a été à moitié légalisé, mais vers l'arrière de sa maison, hélas, laissant les autres habitants de la pente dans l'illégalité.

Observant le plan du quartier, le fondateur de l'Institut d'études urbaines de l'Université nationale de Colombie à Bogotá, Fernando Montenegro, rappelle le développement des villes d'origine hispanique, par la juxtaposition de manzanas (îlots rectangulaires réguliers) et le prolongement de ce tissu urbain (...). Le nouvel arrivant adopte par mimétisme cette forme d'îlot en l'adaptant à ses capacités économiques. Il en résulte un lotissement compact, des rues étroites et des façades sans retrait, des constructions sans étage dans un premier temps, des lots occupés aléatoirement (...). De l'îlot, on passe au quartier et, avec lui, les sentiments d'appropriation et d'identité augmentent chez les occupants. Un mode de transport informel s'installe rapidement, puis l'épicerie arrive comme premier repère, suivie par le dépôt de matériel, la boulangerie, le salon de coiffure, et ainsi de suite, créant le centre des activités et définissant « la » rue principale.

\section{TOURISME EN BIDONVILLE}

David Gómez a fait de sa maison natale de Siloé, à Cali, un petit musée évolutif. Le bidonville est né dans les années 1950, de l'appropriation de terrains d'anciennes haciendas ${ }^{7}$ par des paysans attirés par l'exploi-

7. Grandes exploitations agricoles et leurs locaux d'habitation [NDLR]. 
tation de mines de charbon dans les collines voisines. Ils s'installent en hauteur, habitués aux montagnes et par peur des inondations.

Dans les zones à risques, délaissées après une catastrophe, l'aménagement d'un espace public peut transformer la qualité de vie des habitants proches. C'est le cas du parc mirador « Yo amo a Siloé (j'aime Siloé) ». Sur l'emplacement de maisons rasées par des glissements de terrain meurtriers, devenu terrain de conflits entre bandes de quartier, une succession de terrasses a été édifiée avec des aménagements sportifs et de jeux pour enfants, d'où l'on observe un panorama de toute la ville de Cali (...). Un projet en cours propose l'installation d'un metro-cable (téléphérique) vers Siloé. David souhaite former des jeunes originaires de Siloé au métier de guide touristique, pour expliquer à ceux qui emprunteront les cabines, le temps du trajet, l'histoire du bidonville devenu quartier.

\section{COMME LA VILLE, TOUJOURS EN ÉVOLUTION!}

Veuve et mère de quatre enfants, Rosario $C$. est arrivée très jeune à Cali. Elle y a eu trois autres enfants, dont un, abandonné devant sa porte, qu'elle a adopté. Employée comme femme de ménage, elle complète ses revenus par de petits boulots. Avec ses premières économies, elle achète une baraque dans un bidonville. C'est une pièce sans eau ni électricité, située au bord d'un égout à ciel ouvert, sur un sol en terre, des murs en paille, un toit de tuiles noires en carton. Après quatorze ans, elle finit par bénéficier de la politique d'allocations logement pour la population vulnérable. L'allocation lui octroie une somme d'argent pour l'achat d'une parcelle. Rosario déménage sa baraque, emmenée en morceaux par camion puis remontée sur un nouveau lotissement vide. La nouvelle propriétaire a enfin une adresse postale (...).

Aujourd'hui âgée de 60 ans et toujours femme de ménage à mi-temps, Rosario projette de créer avec sa fille un petit restaurant. Il n'y a plus de commerces dans son quartier, l'insécurité y est prégnante. Elle aimerait déménager et quitter Cali, mais les quelque 25 millions de pesos que vaut sa maison aujourd'hui ne lui offrent pas de grandes perspectives. Un de ses fils habite un logement gratuit proposé par l'État (voir encadré); un autre a refusé d'en bénéficier, il le trouve trop petit et situé dans un quartier qui ne lui plaît pas. Rosario, elle, n’y a pas droit puisqu'elle est déjà propriétaire. 
100000 loggements gुratuits

La Constitution colombienne stipule que l'État s'engage à aider financièrement chaque Colombien pour qu'il puisse accéder à un logement décent (article 51). Le ministère du Logement, de la construction et de l'assainissement est chargé de remettre gratuitement un logement aux familles les plus vulnérables (...). Entre 2012 et 2015, 100000 logements gratuits ont ainsi été remis aux familles. Rares sont pourtant les jugements positifs sur le système, critiqué surtout sur la localisation et la qualité des logements : éloignement du centre ville, des transports en commun et du travail, difficultés de réadaptation pour les familles et d'intégration à un nouvel environnement géographique et humain... Un accompagnement est nécessaire pour expliquer, par exemple, les nouveaux devoirs qu'implique l'accession à la propriété, comme le paiement des factures d'eau et d'électricité. II y a eu des retours volontaires en bidonville ! - CM et LS

\section{«TOUS LES DEUX ANS, LE CHIEN MONTE D’UN ÉTAGE »}

Cette expression de Fernando Montenegro reflète le regard optimiste que nous souhaitons porter sur l'informalité et ses formes d'évolution. Suite à l'auto-construction et aux ressources acquises par une famille, la maison croît d'un étage, et, avec, la terrasse où veille le chien. Une grande part du développement des villes provient de la capacité informelle d'affronter la réalité : celle-ci résout même plus de choses que la formalité! Les bidonvilles ne sont pas des sols inertes, mais au contraire des lieux où les gens s'intègrent rapidement au territoire, ne faisant que consolider des formes de vie qui vont en s'améliorant. Dans le cycle naturel d'une ville, souvent les quartiers riches se dégradent lentement, et, à l'inverse, la qualité de la vie dans les quartiers pauvres progresse graduellement. L'esprit de survie de leurs concepteurs-fabricants-habitants prime. Le savoir-faire propre à cette population « de l'extrême " peut-il interpeller les professionnels de l'urbanisme?

Fruits du travail plus ou moins spontané de l'informalité, les bidonvilles sont un lieu ressource, qui croise densification et étalement, les deux formes de développement de la ville. La collaboration entre habitants et urbanistes devrait permettre de profiter des capacités d'adaptation et de la créativité des pre-

SUR REVUE-PROJET.COM

Retrouvez les articles de Fanny

Gerbeaud, Sylvain Grisot,

Caroline Motta et Lenka Sobotova dans leur version intégrale. miers et du savoir-faire dans la mise en œuvre des seconds. 